\title{
Optimized Segmentation-Adaptive-Based Testing of the Wavelet Co-movement Analysis: the Case of US and G8 Countries
}

\author{
Jitka Poměnková, Eva Klejmová, and Tobiáš Malach \\ Brno University of Technology, Faculty of Electrical Engineering and Communication, Technická 12, 61600 Brno, Czech Republic
}

\begin{abstract}
The paper deals with the identification and the description of the co-movement between the US and G8 countries with regard to the impact of the structural change, i.e. the financial crisis in 2008. For the identification of the co-movement, we use an optimized segmentation-adaptive-based approach (SAB) of significance testing of the power wavelet cross-spectrum. The SAB testing is based on the standard testing for the power wavelet cross-spectrum adapted for the case if the data have several levels of volatility during the time evolution, i.e. the data can be split into several segments with different volatility. The number of segments is set by the heteroscedasticity test and the test for comparing variances in the segments of the time series. The SAB testing allows us to identify significant co-movement with respect to the local variance, which can reveal additional significant co-movement areas. We apply this approach to the monthly data of industrial production index for G8 countries in 1993-2017.
\end{abstract}

\section{Introduction}

Many scientific areas, such as economy, sociology, geoscience etc., analyze co-movements between two time series or signals. An instrument used for the comovement analysis should reflect the area of the application and the nature of data. In the last decade we have seen an increase in the use of advanced methods from technical sciences in social sciences. When using such instruments we need to adapt these methods, to optimize their use and to formulate the correct interpretation of the achieved results. A very useful approach, reflecting both time and frequency behaviour of input time series, are time-frequency (TF) techniques. It is also possible to use time domain techniques or frequency domain techniques separately, however, their ability to capture the frequency behaviour of the analysed time series with respect to the time is rather limited. The well-known TF techniques are Short Term Fourier Transform (FT), TF modification of periodogram, TF varying autoregressive process (TFAR) or continuous wavelet transform (CWT) [1-4].

The co-movement analysis is widely used in the field of economics. If an economic analyst is interested in the time-localisation of the co-movement measure, then wavelets are a very suitable instrument [5], [6]. Many economic applications of the wavelets fall into the area of synchronisation of countries and business cycle analyses [5], globalisation of economies [7], emerging and financial markets analyses [8] etc. Time domain or frequency domain techniques are plausible in this field of research, but do not provide sufficient instruments since they are not able to capture frequency behaviour as a function of the time. On the other hand, the TF approach, i.e. wavelets, propose an examination of comovement of time series via cross spectrum (WCS), cospectrum or cohesion. In this way we can capture a temporal character of co-movement and obtain more precise evaluation of co-movement from time as well as frequency point of view. As many experts agreed, the main advantages of wavelets are: the applicability on stationary and non-stationary time series; the flexibility of choice of mother wavelet with respect to the character of inputs; the ability to uncover unique complicated patterns over time and a good time resolution [4-9].

Pioneer work for statistical significance testing of wavelet power spectrum, the cross-spectrum and the linear coherence is considered to be Torrence and Compo [10] (TC98). Ge [11], [12] (Ge08) improved the work of TC98 by incorporating the sampling period factor in the testing. Both papers work with the same assumption for testing. That is, both wavelet spectra are chi-square distributed with two degrees of freedom for the complex mother wavelet and both time series have the Gaussian white noise (GWN) spectrum. Thus, the square absolute value of its wavelet co-spectrum, i.e. power wavelet co-spectrum (PWCS), can be tested for its significance with respect to the variance of each input time series with the use of the Bessel function.

Thus, the testing of PWCS is a valuable instrument in the assessment of the co-movement of economic indicators which provides measurement of globalisation of economies. A number of economic indicators reflect the development of economy and the changes that occur. The moments of these changes often have the character of structural changes, one of the consequences being a 
change in the volatility of the indicator, such as an oil crisis, financial crisis etc., and thus, it is not difficult to identify them. Then, the assessment of the development of economic indicators or the development of their comovement reflecting these structural breaks, provides valuable information about the globalisation of the world economy.

The paper deals with an assessment of the US and G8 co-movements with regard to the impact of the structural change during the 2008 financial crisis. To identify the co-movement, we propose an optimized segmentationadaptive-based approach (SAB) of the significance testing of the PWCS incorporating the local variance of the data. The SAB testing is based on the standard testing for the PWCS adapted for the case if data are split into several segments with different volatility. The number of segments is set with the help of heteroscedasticity test and the test for comparing variances in the time series segments. To describe the co-movement, we use inverse transform of the identified significant part of PWCS. We apply this approach to monthly data of industrial production index for G8 countries in 1993-2017.

The paper is organized as follows: after the introduction, the Methodology section introduces the theoretical background for significance testing and evaluation of number of segments. In the next section, Application, describes simulated data and summarizes the achieved results. The paper ends with the Conclusion.

\section{Methodology}

In this section we present the theoretical background for the identification of optimum number of segments for the SAB approach of significance testing of the cross spectrum. As the calculation of CWT and the estimation of the PWCS are well-known, we do not describe them. The SAB testing is based on the standard testing approach given by Torrence and Compo [10] and Ge (Ge08) [11], [12]. Therefore, we describe the standard significance test. Consequently, we focus on the description of SAB algorithm for statistical testing and on the criterion for optimum number of segments identification.

\subsection{Standard significance test (STA)}

As written in $\mathrm{Ge08}$, let us consider two independent GWN series $x(t)$ and $y(t)$, with the corresponding variances $\sigma_{\mathrm{x}}{ }^{2}$ and $\sigma_{\mathrm{y}}{ }^{2}$, and wavelet spectras $W_{x}(\mathrm{a}, b)$ and $W_{y}(\mathrm{a}, b)$, where $a$ is the time position and $b$ is the scale. We can define the significance test of its PWCS $\left|C_{x y}(\mathrm{a}, b)\right|^{2}=\left|W_{x}^{*}(\mathrm{a}, b) W_{y}(\mathrm{a}, b)\right|^{2}$

$$
\left|C_{x y}(\mathrm{a}, b)\right|^{2} /\left(\sigma_{\mathrm{x}}^{2} \sigma_{\mathrm{y}}^{2}\right) \sim 0.25 \delta t^{2} W_{2},
$$

where $\delta t$ is the sampling period and $W_{2}$ denotes the probability distribution with a probability density function

$$
f(\mathrm{z})=0.5 \mathrm{~K}_{0}\left(\mathrm{z}^{1 / 2}\right)
$$

$K_{0}(z)$ is here the modified Bessel function of order zero if the complex wavelets are used [13]. The significance of the PWCS can be also tested by the Monte Carlo (MC) simulations, which is based on repeating random sampling.

\subsection{Segmentation-Adaptive-Based testing}

Let us consider the situation that the time series have several volatility changes during the time [16], [17]. We focus on the case when these changes are quick and the moment of the change is quite short, i.e. we did not consider the case when the data have a linearly changing volatility. For such a case we propose the SAB testing approach.

Let us have time series $x$ and $y$ such that one of them or both series have several volatility changes during the time, i.e. is/are heteroscedastic. We are able to identify the moments after which the variance increases or decreases via an expert estimate or by statistical testing [16]. In the case of an economic indicator, the support for the existence of a structural change is an evolution of events in economy of the country. After comparing both series, we can identify the segments (SG) of the time reflecting heteroscedasticity in $x$ and $y$. In each segment we can perform the significance testing according to Ge08. As we described in Klejmová et al. [15] an algorithm for the SAB testing is the following:

- Identification of sub-segments in the time series $\mathrm{x}$, $\mathrm{y}$ according to their variance

- Splitting the total time of duration into the segments for all levels of variance

- Estimation of PWCS

- Significance testing of PWCS with respect to the variance in the segment

\subsection{Identifying the optimum number of segments}

Let us suppose that both time series $x(t)$ and $y(t)$ are heteroscedastic in the time range $t=1, \ldots, T$. Thus, for each series, we are able to identify moments when the data volatility increases or decreases. Consequently, we can determine the corresponding time segments via the identified moments. In some cases it is easy to identify the moments by an expert estimate. In the cases when the assessment of these structural moments in the graphical form of the data presentation is not clear, we can confirm the expected moments via the use of heteroscedasticity test [17].

Let us focus on each series separately. Assume that we applied the test of the equality of variances on the time series $x(t)$ and we identified $n$ moments of volatility changes at $1 \%$ significance level, or $n+k$ at $5 \%$ significance level, or $n+k+l$ at $10 \%$ significance level. Now, we split the time range into $n+k+l$ segments according to all identified time moments for $1 \%$ significance level. In each segment we calculate the variance and we compare the identified variances on the 
basis of standard F-test (test of equality of variances) in $j$ and $j+1$ segment, $j=1, \ldots, n+k+l-1$. Let the null hypothesis hold for the variances in the $j$ and $j+1$ segment

$\mathrm{H}_{0}: \sigma_{i}^{2}={\sigma_{i+1}}^{2}$ (joining of segments), if $S_{j}^{2} / S_{j+1}{ }^{2} \in C I$ where $S_{j}^{2}$ is the sample variance in $j$-th segment, $C I=\left(\mathrm{F}_{\alpha / 2}\left(v_{1}-1, v_{2}-1\right), \mathrm{F}_{1-\alpha / 2}\left(v_{1}-1, v_{2}-1\right)\right)$ is the confidence interval determined by the quantiles of F-distribution with $v_{1}$ degree of freedom in segment $j, v_{2}$ degree of freedom in segment $j+1$ and the risk $\alpha$ is in the predefined difference of approx. max. 1\%. Then, the variances are taken as equal and we connect both segments into one. The minimum measurements in each segment are set to 40 values. As a result we obtain $\mathrm{SG}_{\mathrm{opt}, \mathrm{x}}$ number of segments, $n \leq \mathrm{SG}_{\mathrm{opt}, \mathrm{x}} \leq n+k+l$. After applying the same algorithm on the time series $y(t)$ we obtain $\mathrm{SG}_{\text {opt,y }}$ number of segments.

In the second step, for both time series, we sort the ascending identified moments by time and we focus on the comparison of identified volatility levels between both time series with respect to the identified subsegments. The resultant $\mathrm{SG}_{\mathrm{opt}}$ number of segments split the time range into the $\mathrm{SG}_{\mathrm{opt}}$ sub-segments. If the time series are not heteroscedastic, then we use the whole time range for each series, i.e. $\mathrm{SG}_{\mathrm{opt}}=1$.

\section{Application}

\subsection{Data}

The data set for an empirical analysis consists of the seasonally adjusted monthly data of industrial production index (IPI) from the OECD database for G8 countries (i.e. Canada, Franc, Germany, Italy, Japan, the United Kingdom (UK) and the United States (US)) in the range January 1993 - December 2017 [18]. We examine comovements between the growth cycles of the US and all others G8 countries. The data have been transformed by the first order difference (FOD) of the natural logarithmic values (Fig. 1 right column).

\subsection{Settings of the methods}

For the power wavelet cross-spectrum estimation, we used the complex Morlet wavelet with the central wavelet frequency $f_{0}=1.5$ as the mother wavelet. We set scales corresponding to the range from 0.5 year to 10 -year cycles, with 257 individual wavelet scales. Table 1 presents the selection of optimum number of segments $\left(\mathrm{SG}_{\mathrm{opt}, \mathrm{x}}\right)$ for each country according to the approach described in the methodology and the total number of segments $\left(\mathrm{SG}_{\mathrm{opt}}\right)$ for $\mathrm{SAB}$ testing for the particular pairs (the US and a country).

\subsection{Results}

The empirical analysis consists of seral steps. In the first step we apply the heteroscedasticity test on the time series. After the confirmation of heteroscedasticity we proceed with the identification of optimum number of segments and corresponding time moments for each time series. Then, we split the time range into segments according to the optimum number of segments for the pairs of the time series, i.e. for the US and a G8 country (see Sec. 3.2). In the second step, we estimate PWCS and apply SAB testing for the particular pairs. In the third step, as a supplement, we identify significant comovement via STA approach.

The results of co-movements are presented in Fig. 1 below. We use the following description in all the figures: $x$-axis represents the time, $y$-axis represents frequency measured in the cycles per year and z-axis represents the PWCS. The figures show a twodimensional projection of three-dimensional charts. The intensity of each contour represents the relative importance of the different periodicities and time. The $y$-axis was re-calculated from frequency to the cycles per year to enable better interpretation of economic inputs. Thus, given the monthly character of the data, we denote the sampling frequency $F_{s}$ to be 12 samples per year. The red curve in the figures defines the significant area identified via STA approach, the yellow dashed curve defines the significant area according to SAB testing.

In the case of Canada \& US (Fig. 1), Italy \& US and Russia \& US (Fig. 1) both testing approaches (STA and $\mathrm{SAB}$ ) identify similar significant areas of co-movement. In the case of Canada and Italy we can say that the 2008 crisis was not the only important structural change during the analyzed time contrary to Germany or Japan. In the case of Russia we can see that the 2008 crisis was also an important structural break. Further, in the period 1994-2000 there was a different structural change causing a higher volatility level in this period leading to the extension (1997-2004) of the co-movement area with the US in the cycle of the length 1.5-5 years.

In the case of France \& US (Fig. 1) and UK \& US (Fig. 1) the SAB approach reveals an additional significant area before the 2008 crisis in the cycles of the length of approx. 2.5 years. Specifically, in the case of France in 1994-2004 and in the case of UK in 19952001.

Table 1. Optimal number of segments.

\begin{tabular}{|l|c|l|c|}
\hline \multicolumn{2}{|c|}{$\begin{array}{c}\text { Segments for } \\
\text { each country }\end{array}$} & \multicolumn{2}{c|}{$\begin{array}{c}\text { Total number of } \\
\text { segments }\end{array}$} \\
\hline \multicolumn{2}{|c|}{ SG $_{\text {opt } \mathrm{x} x}$} & \multicolumn{2}{c|}{ SG $_{\text {opt }}$} \\
\hline Canada & 6 & Canada\&US & 7 \\
\hline France & 6 & France\&US & 9 \\
\hline Germany & 4 & Germany\&US & 7 \\
\hline Italy & 4 & Italy\&US & 7 \\
\hline Japan & 4 & Japan\&US & 7 \\
\hline Russia & 4 & Russia\&US & 7 \\
\hline UK & 6 & UK\&US & 9 \\
\hline US & 4 & \multicolumn{2}{|c}{} \\
\cline { 1 - 2 } & &
\end{tabular}

In the case of Germany \& US (Fig. 1) and Japan \& US (Fig. 1) we can see that the significant area, identified via SAB testing compared to the STA, does not cover the cycles of a shorter length during the 2008 crisis. That is, in the case of Germany and Japan, the significant comovement with the US occurs around the 2008 crisis in the cycles of the length $1.5-5$ years. In both these 
countries we can state that the 2008 crisis was the most important structural break during the analyzed time. 


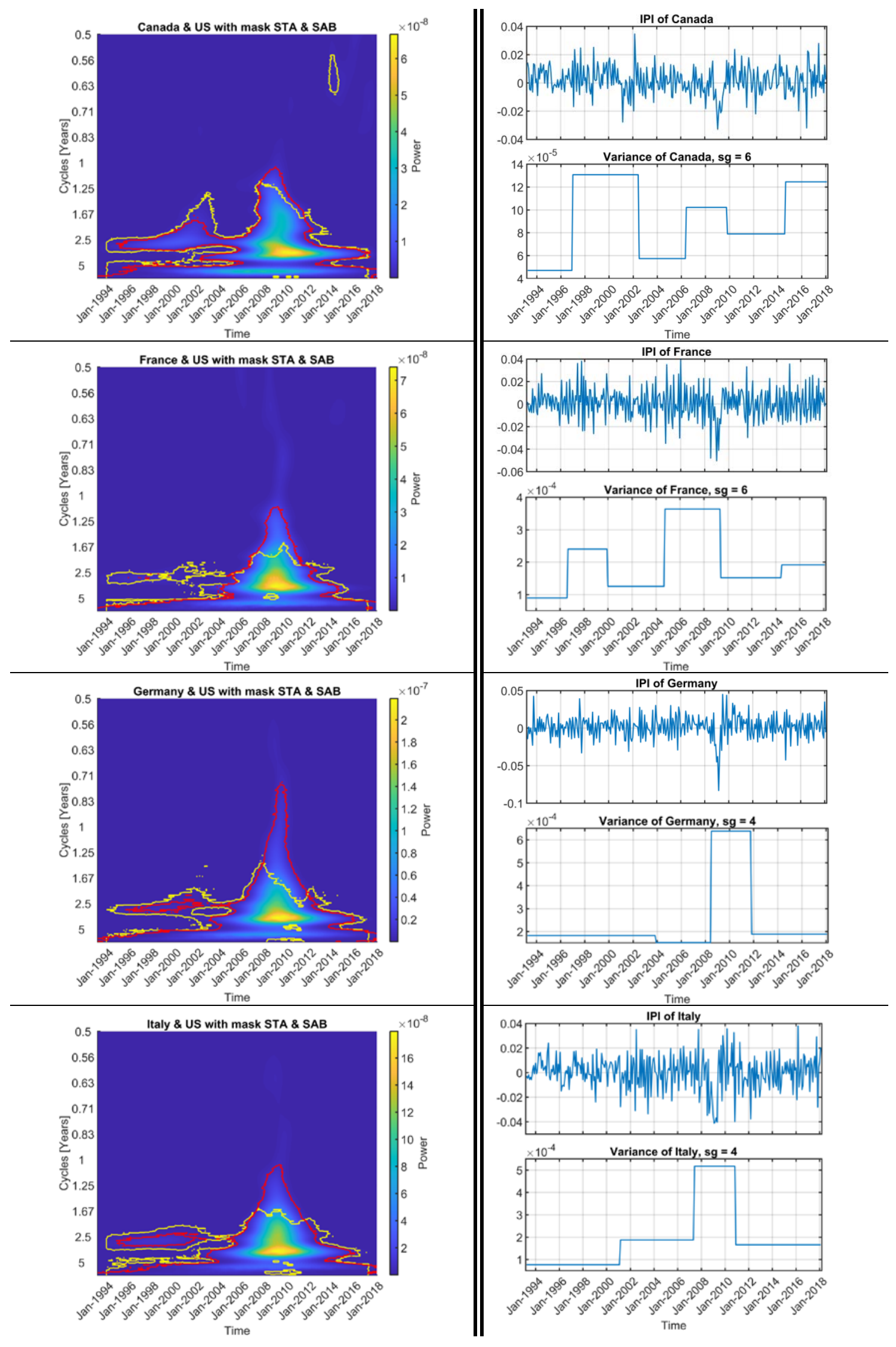




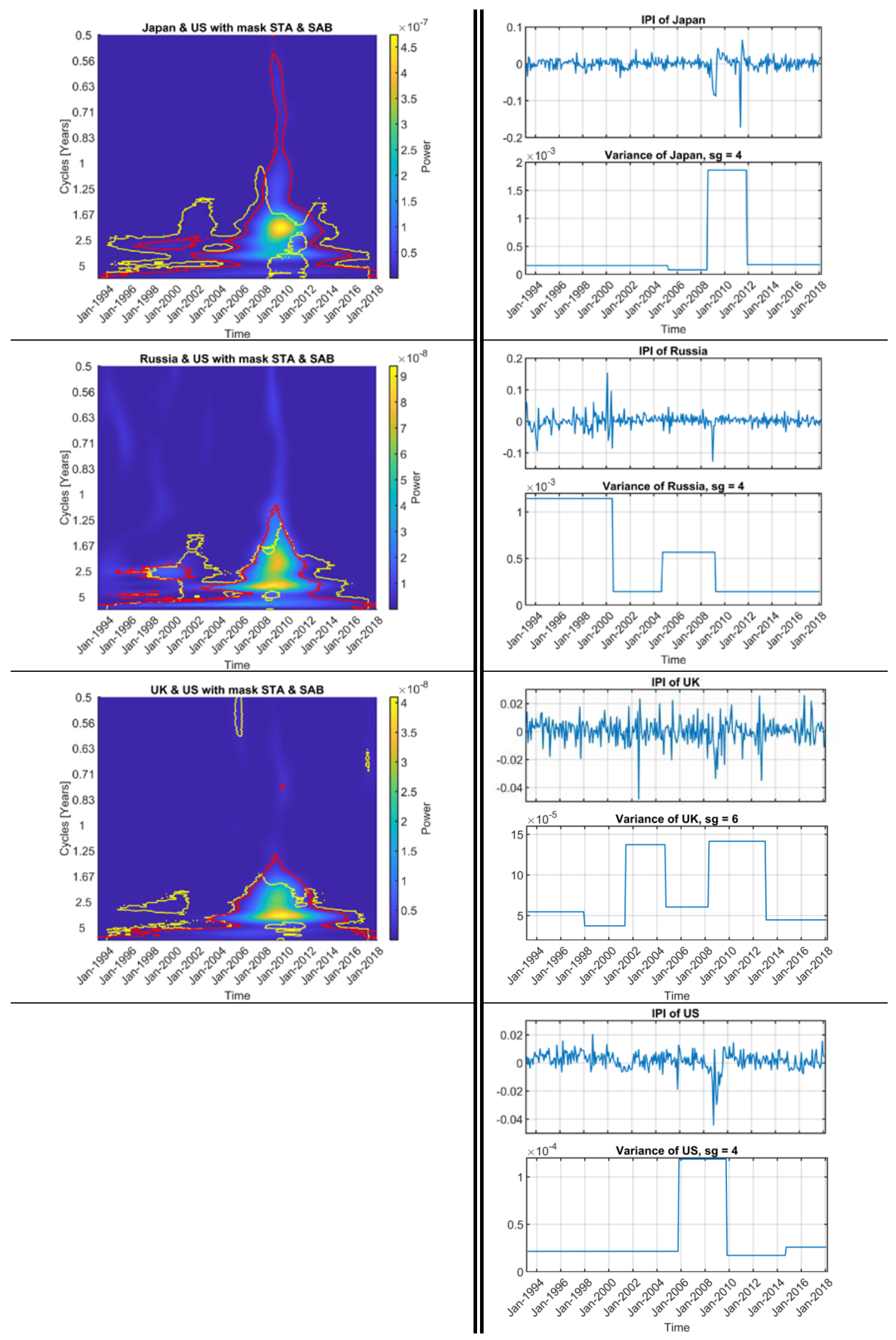

Fig. 1. Co-movement between countries, IPI of each country and variance of their segments. 


\section{Conclusion}

Using real data we assess the co-movements between the US and G8 countries with regard to the impact of the structural change of the financial crisis in 2008. The financial crisis caused a structural break in many economics, which led to the change in volatility level in the economic indicators. We found at least three levels of volatility during the given time range in all analysed time series. Therefore, in the consequent co-movement analysis via PWCS we propose the SAB testing which takes into account the local volatility of the time series. We set the optimum number of segments for co-moved time series and identify significant co-movement areas.

The results confirm the globalisation of the economies and the impact of the 2008 financial crisis on the world economy. In some countries we reveal additional co-movement areas (France, the UK) or the extension of co-movement areas into a longer time period or into an additional cycle length (Japan, Russia). In other countries (Germany, Japan and Italy) we also find that the co-movement in the 2008 crisis was only in the business cycle frequencies, i.e. between the cycles of the length of $1.5-5$ years.

The most energy of the PWCS, i.e. the most important co-movement between US and G8 countries, was identified in the business cycle frequencies. The achieved results can be used for further analyses to look for interconnections between countries co-movement and bilateral trade, in order to reveal additional information about the globalisation and interconnections of economies.

The research described in the paper was supported by the Czech Science Foundation via grant n. 17-24309S and by the Czech Ministry of Education in the frame of National Sustainability Program under grant LO1401. For research, the infrastructure of the SIX Center was used.

\section{References}

1. A. Xu, S. Haykin, R. J. Racine, Multiple window time-frequency distribution and coherence of EEG using slepian sequences and hermite function. IEEE Transactions of Biomedical Engineering, 46 (1999)

2. J. G. Proakis, Ch. M. Rader, F. L. Ling, Ch. L. Nikias, M. Moonen, J. K. Proudler. Algorithms for Statistical Signal Processing. (Prentice Hall, 2002)

3. D. Wang, S. Shilong, W. Tse. A general sequential Monte Carlo method based optimal wavelet filter: A Bayesian approach for extracting bearing fault features. Mechanical Systems and Signal Processing, 52 (2015)

4. W. Jiang, S. Mahadevan, Wavelet spectrum analysis approach to model validation of dynamic systems. Mechanical Systems and Signal Processing, 25 (2011)

5. A. N. Berdiev, Ch-P. Chang, Business cycle synchronization in Asia-Pacific: New evidence from wavelet analysis. Journal of Asia Econ., 37 (2015)
6. Ch. Aloui, B. Hkiri, D. K. Nguyen. Real growth comovement and business cycle synchronization in the GCV countries: Evidence from time-frequency analysis. Economic modelling, 52 (2016)

7. J. Fidrmuc, I. Korhonen, J. Poměnková, Wavelet spectrum analysis of business cycles of China and G7 countries. Applied Economic Letters, 21 (2014)

8. A. K. Tiwari, M. I. Mutascu, A. T. Abulescu, Continuous wavelet transform and rolling correlation of European stock markets. International Review of Economics and Finance, 42 (2016)

9. Z. Ftiti, A. Tiwari, A. Belanés, Tests of financial market contagion: Evolutionary cospectral analysis v.s. wavelet analysis. Computational Economics, 46 (2014)

10. Ch. Torrence, G. P. Compo, A practical guide to wavelet analysis. Bulletin of the American Meteorological society, 79 (1998)

11. Z. Ge, Significance tests for the wavelet cross spectrum and wavelet linear coherence. Annales Geophysicae, 26 (2008)

12. Z. Ge, Significance tests for the wavelet power and the wavelet power spectrum. Annales Geophysicae, 25 (2007)

13. W. T. Wells, R. L. Anderson, J. W. Cell, The distribution of the product of two central or noncentral chi-square varietes, Ann. Math. Stat., 33 (1962)

14. C. P. Robert, G. Casella, Monte Carlo Statistical Methods. (New York: Springer, 2004)

15. E. Klejmová, T. Malach, J. Poměnková, Segmentation based testing of co-movement significance. In : Proceedings of 25th International Conference on Systems, Signals and Image Processing (2018)

16. W. H., Green, Econometric analysis. 7th ed. (Prentice Hall, 2012)

17. D. N. Gujarati, D. C. Porter, Basic Econometrics. $5^{\text {th }} \sim$ ed. (Boston: McGraw-Hill Irwin, 2009)

18. Organisation for Economic Co-operation and Development: National Accounts [online database] (2018). http://stats.oecd.org/index.aspx?DatasetCode=KEI. Accessed 27 March 2018 